\title{
TOXICIDADE DE SEMENTES DE FEDEGOSO (Cassia occidentalis L.) PARA FRANGOS DE CORTE
}

\author{
E. GONZALES \\ Faculdade de Medicina Veterinaria e Zootecnia-UNESP, Cx. P. 502, CEP:18618-000 - Botucatu,SP \\ J.E. BUTOLO \\ SUPRE MAIS - Produlos Qulmicos Lda - C.P. 600 - CEP: 13270-000 - Valinhos, SP \\ R.D. de M. e SILVA; I.U. PACKER \\ Departamento de Zootecnia - ESALQ/USP, C.P. 9 - CEP: 13418-900 - Piracicaba,SP \\ J.M. LAMAS da SILVA $(\dagger)$ \\ Escola de Veterinária - Universidade Federal de Minas Gerais - Belo Horizonte, $M G$
}

\begin{abstract}
RESUMO: Foram realizados três ensaios com o objetivo de se determinar o nível de toxicidade da contaminação de sementes de Cassia occidentalis na alimentação de frangos de corte. Nos dois primeiros ensaios foram utilizadas 640 aves de 1 dia de idade. Os níveis de adição da semente no primeiro ensaio foram, $0 ; 2 ; 4$ e 6\%, obtendo-se 5,77; 84,$62 ; 100$ e $100 \%$ de mortalidade, respectivamente. No segundo ensaio, os níveis utilizados foram $0 ; 0,5 ; 1$ e $2 \%$, obtendo-se $0 ; 3,29 ; 15,73$ e $89,47 \%$ de mortalidade, respectivamente. No terceiro ensaio, utilizou-se 960 aves de 3 dias de idade. Adicionou-se a ração inicial (4-31 dias de idade) e final (32-52 dias) sementes moídas de fedegoso aos níveis de $0 ; 0,1 ; 0,2 ; 0,3 ; 0,4$ e $0,5 \%$. $O$ peso médio final das aves foi 2,$01 ; 1,95 ; 1,95 ; 1,90 ; 1,77$ e $1,58 \mathrm{~kg}$, respectivamente, observando-se diferença significativa $(P<0,05 \%)$ em relação ao controle para 0,$3 ; 0,4$ e $0,5 \%$ de adição. Os valores médios de consumo $(4,33 ; 4,32 ; 4,32 ; 4,28 ; 4,08$ e $3,80 \mathrm{~kg}$, respectivamente) e da conversão alimentar $(2,15 ; 2,21 ; 2,22 ; 2,25 ; 2,31$ e 2,41 , respectivamente) foram significativamente diferentes $(P<0,05 \%)$ para 0,4 e $0,5 \%$ de adição das sementes em relação ao grupo controle. $O$ aspecto histologico de orgãos e tecidos das aves que receberam C.occidentalis, sacrificadas no término das fases inicial e final, foi característico de um processo degenerativo observado no coração, figado, pâncreas, rins, músculo estriado esquelético e intestinos. Os percentuais de mortalidade foram 2,$77 ; 2,08 ; 2,08 ; 0,69 ; 0,69$ e $0 \%$, respectivamente.
\end{abstract}

Descritores: frangos de corte, toxicidade, Cassia occidentalis L., fedegoso.

\section{TOXICITY OF COFFEE WEED (Cassia occidentalis L.) SEEDS TO BROILERS}

\begin{abstract}
ARSTRACT: Three experiments were carried out in order to determine toxic levels of Cassia occidentalis seeds added to broiler feed. On the first two experiments 640 one day-oId sexed broiler chicks were used. The level of inclusion in starter feed of the first trial were $0 ; 2 ; 4$ and $6 \%$ and the mortality rates obtained were $5.77 ; 84.62 ; 100$ and $100 \%$, respectively. In the second trial, levels utilized were $0 ; 0.5 ; 1$ and $2 \%$ and the mortality rates were $0 ; 3.29$; 15.73 and $89.47 \%$, respectively. 9603 -day-old sexed chicks were used in the third experiment. In the starter (4 to 31 days of age) and finisher ( 32 to 52 days) experimental rations the seeds were added at $0 ; 0.1 ; 0.2 ; 0.3 ; 0.4$ and $0.5 \%$. The final body weights were $2.01 ; 1.95 ; 1.95 ; 1.90 ; 1.77$ and $1.58 \mathrm{~kg}$, respectively, being the three highest level groups different from the control. Feed consumption $(4.33 ; 4.32 ; 4.32 ; 4.28 ; 4.08$ and $3.80 \mathrm{~kg}$, respectively) and feed conversion $(2.15 ; 2.21 ; 2.22 ; 2.25 ; 2.31$ and 2.41 , respectively) were significantly different at 0.4 and $0.5 \%$ of seed inclusion comparing to the control group. Histologic aspects of birds that were fed with toxic seeds were characterized by a degenerative process found in the heart, liver, pancreas, kidneys, skeletic muscle and intestines. Mortality rates were $2.77 ; 2.08 ; 2.08 ; 0.69 ; 0.69$ and $0 \%$, respectively.
\end{abstract}

Key Words: broiler, toxicity, Cassia occidentalis L., coffee weed.

\section{INTRODUÇÃo}

. O frango de corte, mesmo em idade de abate, é uma ave em crescimento e, por isso, muito sensível a qualquer condição que perturbe o seu sistema orgânico. Além dos inúmeros fatores prejudiciais ao desenvolvimento das aves, relacionados às condições de doença, manejo e/ou 
deficiênciais nutricionais, a presença dc princípios tóxicos no alimento fornecido aos animais pode se constituir em um fator de desequilibrio orgânico, capaz de levar as aves à morte, se a intoxicação for severa, ou, a um desempenho insatisfatório, se for leve e crônica.

Fedegoso é o nome comum da Cassia occidentalis, uma planta de ciclo anual nativa dos trópicos (BLANCO, 1978). Essa espécie foi assinalada como invasora de culturas de algodão, café, cana-de-acúcar, citros, áreas de pastagens, milho, soja e sorgo (CHARLES, 1975; BLANCO, 1978; COLVIN et al., 1986). A colheita mecânica permite que o milho, a soja e o sorgo utilizados na alimentação animal sejam contaminados com a semente de fedegoso (SIMPSON et al., 1971; COLVIN et al., 1986). Pode-se eliminar a contaminação do milho e da soja através dos procedimentos de limpeza por peneiras comumente utilizados nas unidades de processamento. Porém, para o sorgo, a limpeza, tanto por peneiras como por ventilação, $\varepsilon$ ineficaz já que seus grãos são similares em tamanho e densidade aos do fedegoso (COLVIN et al., 1986).

Verificou-se a ocorrência de miopatias degenerativas dos músculos esqueléticos de bovinos em consequência da ingestão acidental, durante o pastoreio, da planta de C.occidentalis. Os animais afetados apresentavam-se inapetentes, com forte diarréia, ataxia e sinais de disfunção neuromuscular. $O$ achado histopatológico mais comum era de um processo degenerativo dos músculos esqueléticos (DOLLAHITE \& HENSON, 1965, HENSON \& DOLLAHITE, 1966, MERCER et al., 1967, NEAL \& PLUMMER, 1967, PIERCE \& O'HARA, 1967, READ et al., 1968, O'HARA et al., 1970). O quadro clínico e as lesões degenerativas mais graves foram observadas nos animais que ingeriram a semente, considerada a porção da planta potencialmente mais tóxica. Intoxicações experimentais com sementes de fedegoso foram observadas em cavalos, coelhos, aves e suínos, registrando-se mortalidade, que era precedida de um quadro clínico e histopatológico similar ao observado em bovinos (DOLLAHITE \& HENSON, 1965, O'HARA \& PIERCE, 1974a, b).

Nos E.U.A., SIMPSON et al. (1971) relataram alta mortalidade de frangos de corte quando se adicionou sementes de C.occidentalis à rações. A mortalidade aos 21 dias experimentais (28 dias de idade das aves) foram, 65\%, 5\%, $2,5 \%, 2,5 \%$ e $0 \%$ para aves que consumiram ração contendo 4, 2, 1, 0,5 e 0\% da semente. A maioria das aves alimentadas com $4 \%$ das sementes apresentava-se atáxicas ou parcialmente paralizadas antes da ocorrência da morte. Os autores observaram edenıaciação e palidez dos músculos semitendinosos das aves. Estudos histológicos dos músculos revelaram edemaciação focal, fragmentação e necrose das miofibrilas, acompanhadas por proliferação dos núcleos sarcolêmicos.

No Brasil, BUTOLO et al. (1979) observaram que a inclusão de sementes de fedegoso a níveis superiores ou iguais a $1 \%$ na ração de poedeiras leves pode causar mortalidade, paralisação da produção, além de interferir no consumo e peso das aves. A adição das sementes a niveis inferiores a $0,5 \%$ determinou somente queda de produção de ovos. Não há na literatura, pesquisas brasileiras com frangos de corte alimentados com sementes de fedegoso.

O princípio tóxico da C.occidentalis não foi identificado, mas a planta possue componentes potencialmente nocivos como uma albumina, alguns derivados antraquinônicos de forte ação catártica e um alcalóide volátil e termolábil (PULEO, 1966). As folhas e raízes da C.occidentalis contem esses componentes, mas é nas sementes que eles se concentram (PULEO, 1966). O teor dos componentes potencialmente tóxicos das sementes pode variar de acordo com as condições de solo (tipo, adubação, etc) e ambientais (temperatura, umidade) a que a planta foi submetida (ANTON \& DUQUENóIS, 1968, PULEO, 1966). Assim, plantas invasoras de culturas adubadas em regiões tropicais poderiam apresentar sementes com concentrações diferentes dos princípios tóxicos.

0 objetivo do presente trabalho foi avaliar a toxicidade de sementes de fedegoso, obtidas no Estado de São Paulo, para frangos de corte criados em condições brasileiras.

\section{MATERIAIS E MÉTODOS}

Foram realizados três ensaios com frangos de corte. Os dois primeiros experimentos foram conduzidos em caráter exploratório para estabelecer os níveis de adição de sementes de C.occidentalis a ração que não determinassem aumento significativo de mortes para possibilitar o estudo do desempenho das aves em outro ensaio. Em cada um dos dois primeiros experimentos utilizou-se 640 pintos de um dia de idade da linhagem Hubbard alimentados durante 21 dias com ração comercial para a fase inicial de criação à qual se adicionou as sementes moídas em 
concentrações diferentes. Adotou-seo delineamento em blocos ao acaso com 4 tratamentos (níveis de adição da semente $=0,2,4$ e $6 \%$ ) e 4 blocos perfazendo 16 parcelas experimentais de 40 aves (20 machos e 20 fêmeas) cada uma. No ensaio número dois, os níveis de adição da semente moída a ração basal eram $0,0,5,1$ e $2 \%$. Nos dois experimentos foram coletados os dados de peso corporal médio, mortalidade, aspectos clínicos e necroscópicos de aves sacrificadas (4, no ensaio 1 , e 8, no ensaio 2). Para efeito de cálculo do percentual de mortalidade, foram considerados 156 e 152 aves/tratamento nos ensaios 1 e 2 , respectivamente, descontadas as aves sacrificadas para estudos necroscópicos.

No terceiro experimento foram alojados 960 pintos de corte de um dia da linhagem Hubbard em 6 tratamentos e 4 repetições perfazendo 24 unidades experimentais de 40 aves cada (20 machos e 20 fêmeas). Os 6 tratamentos corresponderam à adição de $0,0,1,0,2,0,3,0,4$ e 0,5 de sementes moídas a uma ração basal, inicial e final, balanceadas segundo as exigências apresentadas por SCOTT et al. (1976). O experimento foi dividido em 3 períodos: pré-inicial (1-3 dias de idade das aves), inicial (4 a 31 dias) e final (32 a 52 dias). Na fase pré-inicial foi fornecido somente fubá grosso a todos os tratamentos. Nas demais fases as sementes foram adicionadas às rações inicial e final. Observou-se a mortalidade, o peso, consumo e conversão alimentar. Foram sacrificadas 16 aves de cada tratamento (8 fêmeas e 8 machos) ao final das fases inicial e final para necrópsia e coleta de órgãos. Desse modo, o cálculo do percentual de mortalidade foi feito tendo como base 144 aves por tratamento (160 - 16 aves). Os orgãos e tecidos coletados (cérebro, cerebelo, coração, fígado-vesícula biliar, rins, duodeno e musculatura peitoral e coxal) foram fixados em uma solução de formol a $10 \%$ por período mínimo de $48 \mathrm{~h}$. A técnica de coloração utilizada foi a da hematoxina-eosinas (H.E.) com leitura das lâminas em microscopia ótica.

\section{RESULTADOS E DISCUSSÃo}

Ensaio 1: Decorridos os 21 dias experimentais, os registros de mortes observados foram 5,77, 84,62, 100 e $100 \%$ (TABELA 1) respectivamente, para os níveis de adição de $0,2,4$, e $6 \%$ de sementes de C.occidentalis. Os sinais clínicos apresentados pelas aves tratadas com sementes de C.occidentalis foram: extrema debilidade, desidratação, excrementos diarréicos com colaração esverdeada, tremores, torcicolo, inapetência, permanência ao redor da lâmpada de aquecimento e morte rápida depois dos sintomas de ataxia. As aves sacrificadas aos 7 dias de idade, pertencentes aos tratamentos com 2; 4 e $6 \%$ de adição das sementes de C.occidentalis, apresentaram à necropsia musculatura peitoral, coxal e cardíaca isquêmicas, rins pálidos, fígado congesto, hipertrofia da vesícula biliar e rentenção do saco vitelino. Não se observou conteúdo gastrintestinal, indicando que as aves não se alimentavam.

Ao final do experimento, as únicas aves sobreviventes alimentadas com sementes de C.occidentalis pertenciam ao tratamento com $2 \%$ de adição. Essas aves apresentaram um peso médio de $122 \mathrm{~g}$ em comparação com $529 \mathrm{~g}$ do grupo controle e, a necropsia revelou somente uma leve congestão hepática e retenção do saco vitelino.

$O$ aspecto clínico e necroscópicos foram semelhantes aos referidos por SIMPSON et al. (1971) para frangos de corte. No entanto, os resultados de mortalidade demonstraram a toxidade altamente letal das sementes utilizadas, diferentes daqueles observados por SIMPSON et al. (1971) que encontraram $5 \%$ e $65 \%$ de mortalidade para níveis de adição de 2 e $4 \%$. A discordância sobre a atividade tóxica da $C$.occidentalis pode ser devida à variação nos teores dos princípios tóxicos na semente, dependentes dos fatores locais, nutricionais e fisiológicos da planta (ANTON \& DUQUENÓIS, 1968) ou volatilização do princípio tóxico ocorrida durante o armazenamento ou moagem da semente (PULEO, 1966; BUTOLO et al. 1979).

Ensaio 2: $\mathrm{Na}$ TABELA 2 aparecem o número e percentual de mortes registrados do $1^{\circ}$ ao $21^{\circ}$ dia experimental e o peso médio final por nível de adição. $O$ número de mortes registrado nesse ensaio continuou elevado $(89,5 \%)$ e agudo para o nível de adição de $2 \%$. Esse resultado foi comparável aos $84,62 \%$ de mortes registrado no ensaio anterior e muito diferente do encontrado por SIMPSON et al., (1971), que foi de $5 \%$ para esse nível de adição. Com a inclusão de $1 \%$ das sementes, a mortalidade foi de $15,13 \%$ e caracterizou-se por ter uma evolução sub-aguda, iniciando-se a partir do $10^{\circ}$ dia de administração da semente. Com $0,5 \%$ de adição da semente, registrou-se $3,29 \%$ de mortalidade, não significativo $(\mathrm{P}>0,05) \mathrm{em}$ relação ao grupo controle ( $0 \%$ de mortes). 
TABELA 1 - Peso corporal médio das aves remanescentes, número de mortes e percentual de mortalidade das aves no $21^{\circ}$ dia experimental (Ensaio 1).

\begin{tabular}{lcccc}
\hline & \multicolumn{2}{c}{ Peso Corporal Médio } & \multicolumn{2}{c}{ Mortalidade } \\
\cline { 2 - 5 } \multicolumn{1}{c}{ Tratamentos } & $\mathrm{kg}$ & I.R. $^{1}$ & Número & $\%$ \\
\hline Ração Basal (R.B.) & 0,529 & 100 & $9 / 156$ & 5,77 \\
RB $+2,0 \%$ C. occidentalis & 0,122 & 23,06 & $132 / 156$ & 84,62 \\
RB $+4,0 \%$ C. occidentalis & - & - & $156 / 156$ & 100,00 \\
RB $+6,0 \%$ C. occidentalis & - & - & $156 / 156$ & 100,00 \\
\hline \hline
\end{tabular}

${ }^{1}$ I.R. = Indice Relativo.

TABELA 2 - Peso corporal médio e mortalidade das aves ao final de 24 dias de criação (21 dias experimentais) (Ensaio 2).

\begin{tabular}{lcccc}
\hline & \multicolumn{2}{c}{ Peso Corporal Médio } & \multicolumn{2}{c}{ Mortalidade } \\
\cline { 2 - 5 } \multicolumn{1}{c}{ Tratamentos } & $\mathrm{kg}$ & I.R. ${ }^{1}$ & Número & $\%$ \\
\hline R.B. & 0,529 & 100,00 & $0 / 152$ & $0,0^{\mathbf{a}}$ \\
RB $+0,5 \%$ C. occidentalis & 0,584 & 92,26 & $5 / 152$ & $3,3^{\mathrm{a}}$ \\
RB $+1,0 \%$ C. occidentalis & 0,300 & 47,39 & $23 / 152$ & $15,1^{\mathrm{b}}$ \\
RB $+2,0 \%$ C. occidentalis & 0,132 & 20,85 & $136 / 152$ & $89,5^{\mathrm{c}}$ \\
\hline \hline
\end{tabular}

${ }^{1}$ I.R. = Indice Relativo.

$a, b, c,=$ Tratamentos com diferentes letras, diferem significativamente $(P \leq 0,05)$ entre si.

O quadro clínico das aves sobreviventes no tratamento com $2 \%$ de adição foi idêntico ao referido no ensaio número 1 e apresentaram um peso médio final de $132 \mathrm{~g}$. As aves do tratamento com $1 \%$ de adição também se apresentaram debilitadas, porém eram mais ativas que as do tratamento superior. Não se evidenciou fezes diarréicas nesse tratamento.

O peso médio das aves sobreviventes desse tratamento foi $300 \mathrm{~g}$. As aves do tratamento com $0,5 \%$ de adição embora mostrassem menor peso $(584 \mathrm{~g})$ em relação ao grupo controle $(633 \mathrm{~g})$, aparentemente apresentavam-se sadias.

Sci. agric., Piracicaba, 51(1):169-174,jan./abr., 1994
Ensaio 3: Na TABELA 3 são mostrados os valores médios de peso, consumo alimentar e conversão alimentar e mortalidade observados nesse ensaio ao final do período experimental (49 dias experimentais ou 52 dias de idade das aves). A análise do peso corporal médio das aves revelou diferenças significativas $(P<0,05)$ para os níveis de adição igual ou superior a $0,3 \%$, indicando o efeito depressor de peso da adição da semente às rações. O consumo e a conversão alimentar foram estatisticamente piores $(\mathrm{P}<0,05)$ para os grupos que receberam os dois maiores níveis de sementes moídas. 
TABELA 3 - Dados médios finais (52 dias de idade) de peso corporal, consumo, conversão alimentar e mortalidade de frangos de corte alimentados com sementes moídas de C. occidentalis (Ensaio 3).

\begin{tabular}{|c|c|c|c|c|c|c|c|}
\hline \multirow[b]{2}{*}{ Tratamentos } & \multicolumn{2}{|c|}{ Peso Corporal } & \multicolumn{2}{|c|}{ Consumo Alimentar } & \multicolumn{2}{|c|}{ Conversão Alimentar } & \multirow{2}{*}{$\begin{array}{c}\text { Mortalidade } \\
\% \\
\end{array}$} \\
\hline & kg & IR & kg & IR & kg:kg & IR & \\
\hline R.B. & $2,01^{\circ}$ & 100 & $4,33^{2}$ & 100 & $2,154^{\circ}$ & 100 & 2,77 \\
\hline $\mathrm{RB}+0,1 \%$ C. $o$. & $1,95^{\mathrm{ab}}$ & 97,16 & $4,32^{\mathrm{a}}$ & 99,72 & $2,211^{\mathrm{a}}$ & 97,42 & 2,08 \\
\hline $\mathrm{RB}+0,2 \%$ C.o. & $1,95^{\mathrm{ab}}$ & 97,11 & $4,32^{:}$ & 99,84 & $2,215^{a}$ & 97,25 & 2,08 \\
\hline $\mathrm{RB}+0,3 \%$ C.o. & $1,90^{\mathrm{b}}$ & 94,68 & $4,28^{a}$ & 98,75 & $2,248^{\mathrm{ab}}$ & 95,82 & 0,69 \\
\hline $\mathrm{RB}+0,4 \%$ C.o. & $1,77^{\circ}$ & 87,81 & $4,08^{b}$ & 94,09 & $2,309^{b}$ & 93,29 & 0,69 \\
\hline $\mathrm{RB}+0,5 \%$ C.o. & $1,58^{\mathrm{d}}$ & 78,51 & $3,80^{c}$ & 87,74 & $2,410^{c}$ & 89,38 & 0,00 \\
\hline
\end{tabular}

$\mathrm{a,b,c,d}=$ Médias dos tratamentos com diferentes letras na mesma coluna diferem significativamente $(P \leq 0,05)$

$O$ resultado de mortalidade observado durante todo o período experimental demonstrou a influência não letal da adição das sementes de C.occidentalis nos níveis estudados.

Não se observou sintomatologia clínica de intoxicação e nem lesões macroscópicas nas aves alimentadas com sementes de $C$.occidentalis nesse ensaio. Os aspectos histopatológicos das lesões das aves sacrificadas aos 28 dias experimentais não diferiram nas parcelas que receberam as sementes moídas de C.occidentalis. As aves apresentaram um quadro caracterizado basicamente por processo regressivo variável de intensidade, de indivíduo para indivíduo e de tratamento para tratamento. Assim, pode-se descrever as alterações microscópicas sem destacar o tratamento a que pertencem, pois não houveram variações marcantes entre os tratamentos. Os seguintes quadros histológicos foram observados:

Coração: degeneração turva de fibras musculares cardíacas, apresentando algumas vezes fragmentação das mesmas. Infiltrações discretas de células mononucleares linfoplasmocitárias nos interstícios celulares.

Figado: Degeneração turva, esteatose e necrose de coagulação associada a dissociação de lâminas hepáticas. Esporadicamente verificou-se, também, focos hiperplásicos de folículos linfóides.
Pâncreas: Degeneração turva e necrose de coagulação dos ácinos glandulares.

Intestinos: hipotrofia do epitélio da mucosa intestinal com sensível redução da espessura da mesma. Os folículos linfóides eram escassos e hipotróficos. Havia, também, menor fluxo sanguíneo no estroma conjuntivo.

Rins: degeneração turva do epitélio tubular com acúmulo de material no lúmen, formando cilindros hialinos.

Adrenal: Discreta hiperplasia de células da camada cortical.

Músculo estriado esquelético: Necrose de coagulação focal, abrangendo grupos de fibras musculares. $\mathrm{Na}$ área de necrose, observou-se discreta infiltração de células mononucleares linfócitos.

No cérebro, baço e bolsa de Fabrício não se encontraram alterações microscópicas dignas de nota.

O quadro histopatologico apresentado pelas aves sacrificadas aos 52 dias de idade que receberam C.occidentalis, caracterizou-se por lesões predominantemente regressivas, semelhantes às descritas anteriormente. 
Entretanto, as alterações eram menos extensas e de característica mais discreta, muitas delas com sinais evidentes de regeneração, principalmente a nível hepático, renal e intestinal. Os achados histológicos indicaram uma tendência à evolução de C.occidentalis quando presente à ração em níveis inferiores a $0,5 \%$.

\section{CONCLUSÃO}

Nas condições experimentais assinaladas, os resultados indicaram a ação tóxica das sementes de Cassia occidentalis para frangos de corte, interferindo no desempenho das aves nos níveis superiores a $0,3 \%$ de adição e determinando sintomatologia clínica de intoxicação e mortalidade em níveis superiores a $0,5 \%$ de adição.

\section{REFERENCIAS BIBLIOGRÁFICAS}

ANTON, R.; DUQUENOIS, P. Contribuition a l'étude chimique du Cassia occidentalis L. Annales Pharmacentiques Françaises, Paris, v.26, n.11, p.673-680, 1968.

BLANCO, H.C. Catálogo das espécies de mato infestantes das áceas cultivadas no Brasil - Família do amendoim do campo (Leguminosae). O Biologico, São Paulo, v.44, p.33-90, 1978.

BUTOLO, J.E.; SILVA, J.M.J. da; NERY, J.R. Efeitos da Cassia occidentalis (fedegoso) em poedeiras comerciais quando em produção. In: CONGRESSO BRASILEIRO DE AVICULTURA, 6., Belo Horizonte, 1979. Anais ... Belo Horizonte, 1979. p.352-367.

COLVIN, B.M.; HARRISON, L.R.; SANGSTER, L.T. Cassia occidentalis toxicosis in growing pigs. Journal of the American Veterinary Medical Association, Chicago, v.189, n.4, p.423-426, 1986.

DOLLAHITE, J.W.; HENSON, J.B. Toxic plants as the etiologic agent of myopaties in animal. American Journal of Veterinary Research, Chicago, v.26, p.749-752, 1965.

HENSON, J.B.; DOLLAHITE, J.W. Toxic myo degeneration in calves produced by experimental Cassia occidentalis intoxication. American Journal of Veterinary Research, Chicago, v.27, p.947-949, 1966.

Sci. agric., Piracicaba, 51(1):169-174, jan./abr., 1994
MERCER, H.D.; NEAL, F.C.; HDMES, J.A. Cassia occidentalis toxicosis in cattle. Journal of the American Veterinary Medical Association, Chicago, v.151, p.735-741, 1967.

NEAL, F.C.; PLUMMER, C.B. Cassia occidentalis poisoning in cattle. Annual Report of the Forida Agricultural Experiment Station, Gainesville, p.207, 1967.

O'HARA, P.J.; PIERCE, K.R. A toxic cardiomyopathy caused by Cassia occidentalis. I. Morphologic studies in poisoned rabbits. Veterinary Pathology, New York, v.11, n.2, p.97-109, 1974a.

O'HARA, P.J.; PIERCE, K.R. A toxic cardiomyopathy caused by Cassia occidentalis. II. Biochemical studies in poisoned rabbits. Veterinary Pathology, New York, v.11, n.2, p.110-124, 1974 b.

O'HARA, P.J.; PIERCE, K.R.; READ, W.K. Effects of vitamin $E$ and selenium on Cassia occidentalis intoxication in cattle. American Joumal of Veterinary Research, Chicago, v.31, n.12, p.2151-2156, 1970.

PIERCE, K.R.; O'HARA, P.J. Toxic myopathy in Texas cattle (Cassia occidentalis poisoning). Southwestern Veterinarian, College Station, v.20, p.179-183, 1967.

PULEO, L.E. Isolation of an alkaloid from Cassia occidentalis. Thesis (M.S.) - Texas A \& M. University, College Station, 1966. 26p.

READ, W.; KAY, K.; PIERCE, K.R. Ultrastrutural lesions of an acute toxic cardiomyopath of cattle. Laboratory Investigations, New York, v.18, p.227-231, 1968.

SIMPSON, C.F.; DAMRON, B.L.; HARMS, R.H. Toxic myopathy of chicks fed Cassia occidentalis seeds. Avian Diseases, Ithaca, v.15, p.284-90, 1971.

Enviado para publicação em 30.07 .93

Aceito para publicação em 08.09 .93 